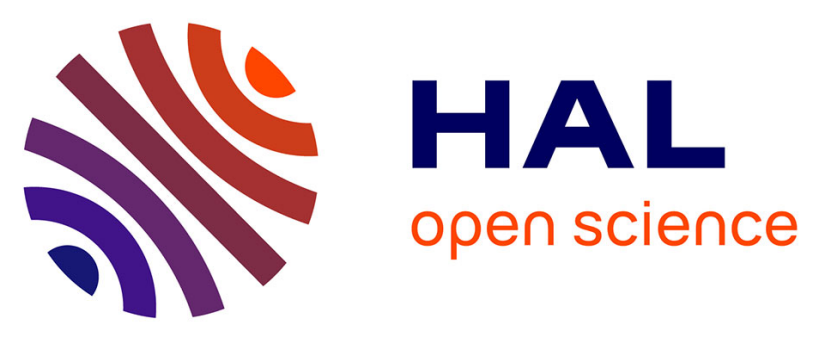

\title{
Porosity-driven large amplitude dynamics for nitroaromatic sensing with fluorescent films of alternating $\mathrm{D}-\pi$-A molecules
}

Jean-Pierre Malval, Marion Cranney, Sylvain Achelle, Huriye Akdas-Kiliç, Jean-Luc Fillaut, Nolwenn Cabon, Françoise Robin-Le Guen, Olivier Soppera, Yann Molard

\section{To cite this version:}

Jean-Pierre Malval, Marion Cranney, Sylvain Achelle, Huriye Akdas-Kiliç, Jean-Luc Fillaut, et al.. Porosity-driven large amplitude dynamics for nitroaromatic sensing with fluorescent films of alternating D- $\pi$-A molecules. Chemical Communications, 2019, 55 (95), pp.14331-14334. 10.1039/c9cc07227f . hal-02438559

HAL Id: hal-02438559

https://hal-univ-rennes1.archives-ouvertes.fr/hal-02438559

Submitted on 18 Feb 2020

HAL is a multi-disciplinary open access archive for the deposit and dissemination of scientific research documents, whether they are published or not. The documents may come from teaching and research institutions in France or abroad, or from public or private research centers.
L'archive ouverte pluridisciplinaire HAL, est destinée au dépôt et à la diffusion de documents scientifiques de niveau recherche, publiés ou non, émanant des établissements d'enseignement et de recherche français ou étrangers, des laboratoires publics ou privés. 


\title{
Porosity-driven Large Amplitude Dynamics for Nitroaromatics Sensing with Fluorescent Films of Alternated D- $\pi$-A Molecules
}

\author{
Jean-Pierre Malval*1, Marion Cranney ${ }^{1}$, Sylvain Achelle ${ }^{* 2}$, Huriye Akdas-Kiliç ${ }^{2}$, Jean-Luc Fillaut ${ }^{2}$,
} Nolwenn Cabon ${ }^{2}$, Françoise Robin-le Guen ${ }^{2}$, Olivier Soppera ${ }^{1}$, Yann Molard ${ }^{2}$.

We report herein the structural properties and nitroaromatics sensing performances of fluorescent thin films formed by alternated donor-acceptor $\pi$-conjugated chromophores. The incorporation of a flexible one-dimensional alkyl chain in the chromophore backbone drastically accelerates by more than one order of magnitude the real-time dynamics fluorescence sensing toward 2,4-dinitrotoluene (DNT) vapors.

The detection of nitroaromatic explosives constitutes a strategic concern in the context of homeland security and environment protection. A large panel of analytical techniques going from expensive and sophisticated instrumentations to low-cost portable detectors is currently available for this purpose. Of particular interest, fluorescent vapor-phase sensors have been extensively developed ${ }^{1-6}$ in concomitance with the emergence of a huge variety of fluorescent sensing materials such as conjugated organic/inorganic polymers ${ }^{2}, 7$, dendrimers ${ }^{8-10}$, molecular imprinted polymers ${ }^{11}$, supramolecular 3D-architectures ${ }^{12}$. Small fluorescent molecules ${ }^{1}$ are also attractive since they constitute relevant building block motifs for self-assembly thin-film sensors. In this domain, one challenging issue relies in controlling at molecular scale the directionality of structural interactions ${ }^{13,14}$ to optimize the macromolecular film porosity which drives the sensor time response with the maintain of a reasonable fluorescence emission in solid state which guarantees the sensor sensitivity.

In line with this objective, we present herein the DNT sensing performances of fluorescent thin-films made of alternated triphenylamine-pyrimidine chromophores (Scheme 1) whose $V$-shaped $\pi$-conjugated structure is initially configured to promote cofacial self-packing. The structural introduction of a long flexible alkyl chain into a judicious position of the chromophore backbone hardly affects the electronic and the photophysical properties but drastically impacts the DNT-vapor sensing dynamics.

${ }^{1}$ Institut de Science des Matériaux de Mulhouse CNRS-UMR 7361, Université de Haute Alsace, 15 rue Jean Starcky, 68057, Mulhouse, France.

2 Université Rennes, Institut des Sciences Chimiques de Rennes CNRS UMR 6226, F-35000 Rennes.

E-Mails: jean-pierre.malval@uha.fr and sylvain.achelle@univ-rennes1.fr

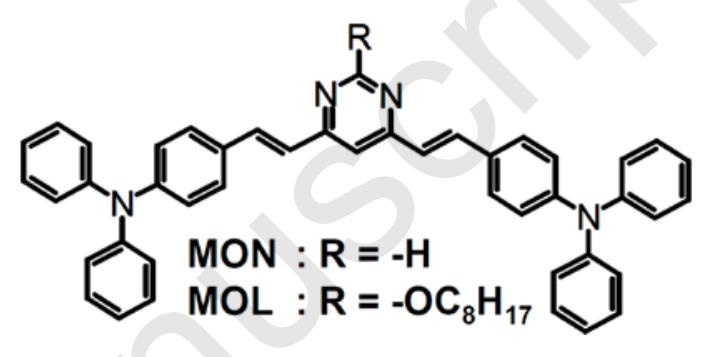

Scheme 1. Molecular structures of the chromophores.

The normalized absorption and fluorescence spectra in toluene and spin-coated films are shown for both chromophores in Figure 1. The spectroscopic data are reported in Tables S1-S2. In liquid medium, MON and MOL both exhibit the same photophysical fingerprint. Their longest wavelength absorption bands which are located in the $380-450 \mathrm{~nm}$ range have $\varepsilon_{\text {MAX }}$ of the same magnitude ( $\left.48000 \mathrm{M}^{-1} \mathrm{~cm}^{-1}\right)$. This band corresponds to a strongly allowed $S_{0} \rightarrow S_{1}$ transition implying a long range charge delocalization from the external triphenyl amine moieties (donors) to the pyrimidine core (acceptor) ${ }^{15,16}$.

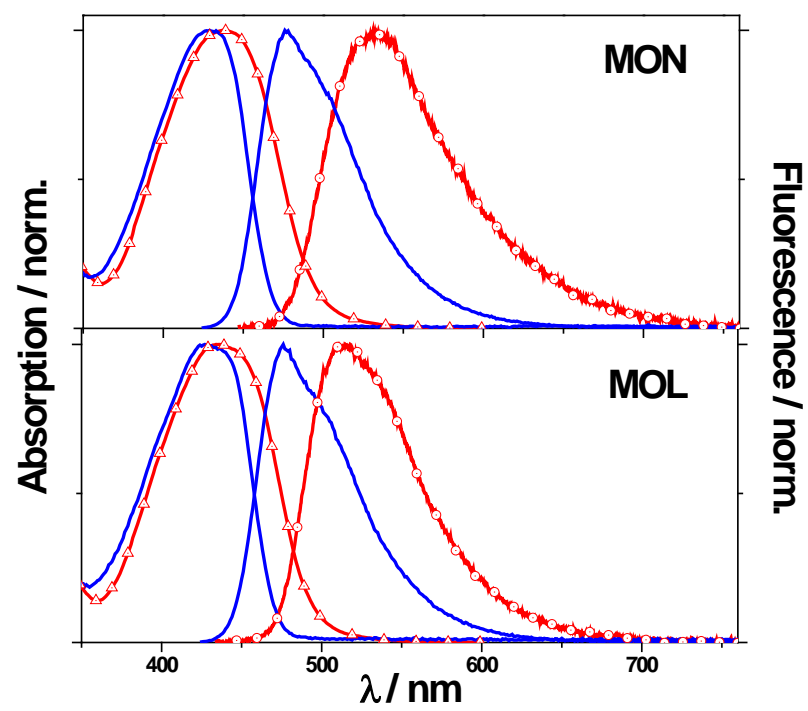

Fig.1 Normalized absorption and fluorescence spectra of compounds in toluene and in spin-cast film (absorption: triangles and fluorescence: circles). 
In liquid medium, these chromophores are strongly emissive with fluorescence quantum yields $\left(\Phi_{f}\right)$ higher than 0.6 whatever the solvent polarity (Table S2). The radiative rate constant $\left(\mathrm{k}_{r}\right)$ remains also invariant indicating that the fluorescence deactivation process occurs via a relaxed $S_{1}$ state with the same electronic configuration in both cases. In addition, the fluorescence bands undergo a strong red shift $\left(\Delta v_{f l u o}\right)$ by about $4300 \mathrm{~cm}^{-1}$ on going from toluene to acetonitrile. Such solvatochromic effects is in line with the enhancement of the $S_{1}$ state dipole moment with respect to that of $S_{0}$ one. This high emissivity associated with a strong charge transfer at $S_{1}$ state clearly indicates the occurrence the radiative deactivation from an intramolecular charge transfer (1ICT) state with a typical planar geometry. In thin films, the dense molecular organization in solid state combined to the inherent heterogeneous environment around the emitting species open up new radiationless routes such aggregation-caused quenching ${ }^{17}, 18$ which competes with the ${ }^{1} \mathrm{ICT}$ state radiative deactivation. As a consequence, the fluorescence time decays of the films become non exponential as compared to those measured in liquid solution (see Figure S1). The averaged emission lifetimes in solid state are divided by a factor $\sim 2$ for both compounds with corresponding fluorescence quantum yields decreasing to values close to 0.2 (Table S1). In parallel, both absorption and fluorescence spectra are redshifted on going from toluene to spin-cast film (see Figure 1). In order to probe the polarity environment around the emitting species in solid state, the maximum fluorescence wavenumbers $\left(v_{f}^{\mathrm{MAX}}\right)$ in thin-films have been reported on the solvatochromatic plots for each chromophore as shown in Figure S2. These solvatochromic plots are obtained by correlating $v_{\text {fluo }} \mathrm{MAX}$ of the dyes diluted in various solvents with the corresponding Lippert-Mataga polarity function ${ }^{19,} 20(\Delta \mathrm{f})$. According to this methodology, it can be inferred that the emitting species in thin films experience a surrounding environment with an estimated $\Delta f$ of about 0.27 and 0.23 for MON and MOL respectively. Such a significant difference in the polarity function clearly denotes a more polar environment for MON molecules as compared to MOL ones which should be ascribed to a more compact molecular organization for MON film with a subsequent enhancement of dipolar interactions. Indirectly this structural effect should preclude a change in film porosity on going from MON to MOL. It is clear that the long alkyl side chain of MOL contributes to enlarge the intermolecular spacing and thereby promotes a larger free volume within the thin-film. In order to illustrate such a structural effect, the self-assembly properties of the chromophores have been investigated by scanning tunneling microscopy (STM) in 1-phenyloctane on highly oriented pyrolytic graphite (HOPG) surfaces. Figure 2 depicts STM topographic images of self-assembled layers of MON and MOL at the solid-liquid interface.

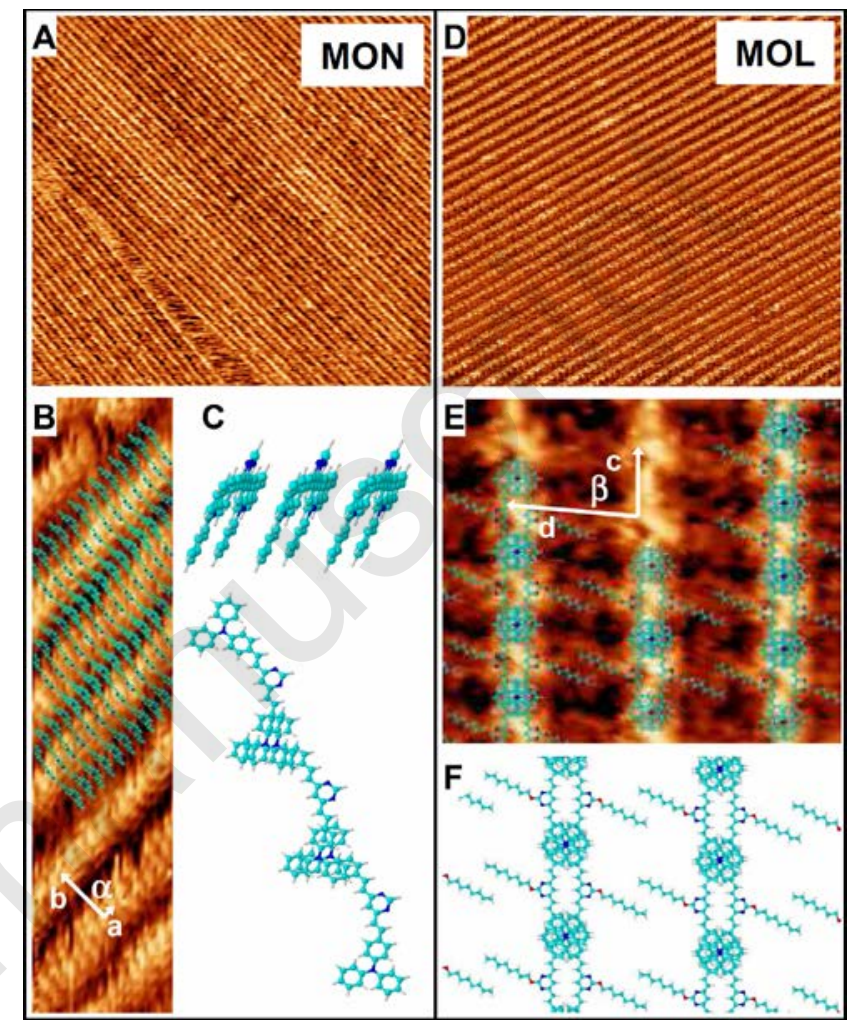

Fig.2 Topographic STM images at 1-phenyloctane-HOPG interface of molecular self-assembled layers. MON: A. $(89.6 \mathrm{~nm} \times 83.3 \mathrm{~nm}$; $-1.0 \mathrm{~V})$; B. (4 nm x $17.6 \mathrm{~nm},-1.0 \mathrm{~V}$ ); C. Possible self-assembly model (up: side view, down: front view). MOL: D. (89.6 nm $\times 83.3 \mathrm{~nm}$; $1.5 \mathrm{~V})$; E. (8.8 nm x $7.6 \mathrm{~nm},-1.5 \mathrm{~V})$; F. Possible self-assembly model.

Two distinctive self-organizations are observed. In the case of MON, the molecules self-assemble in compact domains composed of parallel rows with an oblique lattice. Using self-correlation measurements, we identify its mean unitcell parameters $\mathrm{a}=0.42 \pm 0.02 \mathrm{~nm}, \mathrm{~b}=1.77 \pm 0.04 \mathrm{~nm}$ and $\alpha$ $=88 \pm 2^{\circ}$, leading to an area of $0.74 \mathrm{~nm}^{2}$ per molecule. These values indicate that MON molecules are in edge-on conformation with their long axis parallel to the substrate, probably slightly tilted from the direction perpendicular to the surface. On the basis of the model shown in Figure $\mathbf{2 C}$, $\pi-\pi$ stacking implying the entire aromatic structure of MON constitutes the main interaction that governs the observed self-organization. In the case of MOL, the molecules also self-assemble in domains composed of parallel lines with an oblique lattice. By contrast to MON, MOL molecules are lying flat on the substrate with the pyrimidine cores completely exposed. The parameters of the unit-cell are $\mathrm{c}=$ $1.55 \pm 0.02 \mathrm{~nm}, \mathrm{~d}=2.95 \pm 0.02 \mathrm{~nm}$ and $\beta=83 \pm 2^{\circ}$ which leads to an area of $2.27 \mathrm{~nm}^{2}$ per molecule. It is noteworthy that this area is $\sim 3$ times larger than that measured for MON. Based on the model shown in Figure 2F, we may 
assume that the intermolecular interactions governing the self-assembly of MOL molecules result from a combination of $\pi-\pi$ stacking interactions between superimposed triphenylamine groups as well as Van der Waals interactions between the partially interdigitated alkyl chains. These latter intermolecular repulsive interactions will strongly influence the macromolecular porosity in thinfilm configuration with subsequent effects on the time detection of DNT vapors as will be developed hereafter. In liquid medium, the presence of DNT leads to a dynamic quenching of the chromophores emission. Both fluorescence intensities and emission lifetimes decrease upon addition of DNT leading to Stern-Volmer constants of ca. 48 and $44 \mathrm{M}^{-1}$ for MON and MOL respectively in acetonitrile (see Figure S3). This quenching process typically proceeds through a photoinduced electron transfer (PeT) from the singlet excited state of the chromophore (donor) to DNT (acceptor). Consecutively, a back electron (BeT) should regenerate the chromophore to ground state. However, it is possible to observe an effective photo oxidation of the dyes in presence of a large excess of DNT. Figure S4A shows the evolution of the absorption spectrum of an irradiated ACN solution of MOL in presence of 100 eq. of DNT. While the absorption spectrum of MOL without DNT is relatively invariant upon irradiation (Figure S4B), the presence of DNT clearly triggers important spectral changes. The main absorption band of MOL collapses in concomitance with the growth of a new band in 500-650 $\mathrm{nm}$ range. This band corresponds to the pyrimidine protonated form of MOL since it can be neutralized by addition of a potassium hydroxide ACN solution. This acid form corresponds in fact to a by-product consecutive to the production of protons due to the dimerization of $\mathrm{MOL}$ radical cations (MOL*+). Indeed, it is well established that the one-electron oxidation of partially substituted triphenylamine derivatives generates unstable radical cations which undergo dimerization in the para position to the nitrogen centers ${ }^{21-23}$. In order to confirm the occurrence of these sequencing reactions, cyclic voltammetry coupled with spectroelectrochemical measurements have been performed. First, it should be noted that both MOL and MON present similar oxidation potentials whose values are gathered in Table S3. The cyclic voltammogram of MOL which is displayed in Figure $\mathbf{3 B}$ shows a first oxidation wave at $0.88 \mathrm{~V}$ vs. SCE. This irreversible wave which is only observed during the first forward scan corresponds to the oxidation of $\mathrm{MOL}$ to $\mathrm{MOL}^{\bullet+}$. The second oxidation wave at higher potential ( $\mathrm{E}_{o x^{2}} \sim 1.09 \mathrm{~V} v \mathrm{vs}$. SCE) should be ascribed to the oxidation of the follow-up product (i.e. dimer). Figure 3A depicts the spectroelectrochemical spectrum of MOL in $\mathrm{ACN}$ and its time dependent absorption changes upon applying a constant potential corresponding to the first oxidation reaction. Interestingly, the spectral changes are comparable to those previously observed for the photo oxidation of MOL in presence of DNT with the difference that an additional band is growing in the $600-900 \mathrm{~nm}$ range. This band should presumably correspond to the dimer product which is generated in larger yield as compared to that of the photo oxidation reaction with DNT.

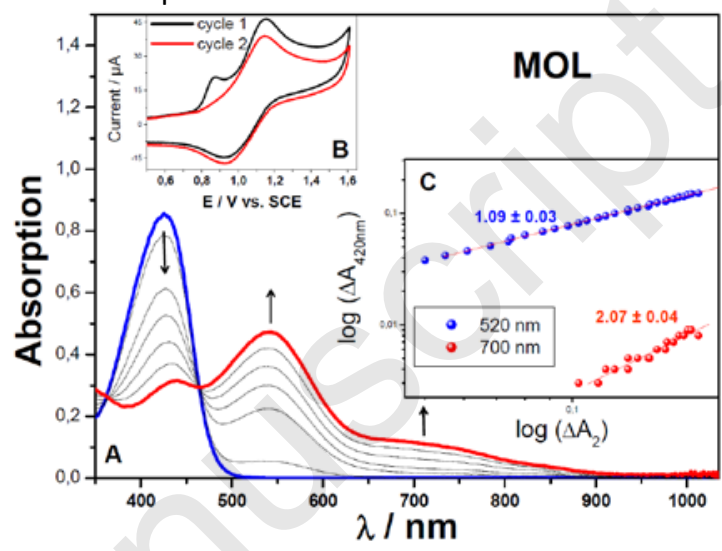

Fig.3 A. Time-dependent changes of the absorption spectrum of MOL upon the first oxidation reaction at constant potential of 0.85 V / SCE. B. CVs of MOL oxidation in ACN $(0.18 \mathrm{mM}$ MOL, $0.1 \mathrm{M}$ TBAPF $\left._{6}, 100 \mathrm{mV} \mathrm{s}^{-1}\right)$. C. Log-log plots of $\Delta \mathrm{A}_{420 \mathrm{~nm}}$ vs. $\Delta \mathrm{A}_{520 \mathrm{~nm}}$ and $\Delta \mathrm{A}_{420 \mathrm{~nm}}$ Vs. $\Delta \mathrm{A}_{700 \mathrm{~nm}}\left(\Delta \mathrm{A}_{\lambda i}\right.$ are in absolute values).

In Figure 3C, the time-dependent absorption changes of MOL at $420 \mathrm{~nm}$ have been correlated to those of the products absorbing at $520 \mathrm{~nm}$ and $700 \mathrm{~nm}$ respectively. Whereas a first-order kinetics reaction is derived for the production reaction of the acid form at $520 \mathrm{~nm}$, a secondorder kinetics is clearly observed for the long-wavelength absorbing product which is consistent with a dimer production. Hence in liquid medium, the presence of DNT promotes a dynamic fluorescence quenching and, in a less extent, a photoinduced oxidation of the chromophores. In solid-state, the response of the molecule films exposed to DNT vapors mainly corresponds to an oxidative fluorescence quenching. To illustrate this effect Figure S5 displays several epifluorescence images of MOL thin-film before and after exposure to DNT vapors. As a reference experiment, the image $(a)$ shows the fluorescence of a film which was excited at $365 \mathrm{~nm}$ during 5 min under $\mathrm{N}_{2}$ stream. No photobleaching was observed demonstrating the film photostability. However, when the same film is irradiated with introduction of DNT vapors, a strong decrease of the film epifluorescence is observed after $15 \mathrm{~s}$ excitation as shown in image (b). The corresponding absorption band of MOL (see Figure S5C) clearly collapses which confirms the photo oxidation process. In this latter case, it should be emphasized that we did not detect the absorption bands of the by-products presumably because their subsequent generation which is diffusion-controlled is strongly hampered in solid state. Hence, it is clear that the analyte detection for both chromophores occurs through a DNT- 
triggered oxidative fluorescence sensing. However, depending on the nature of the chromophore, the real-time sensing dynamics are strikingly different. Figure $\mathbf{4}$ displays the kinetics for fluorescence quenching of MON and MOL films exposed to low vapor pressure of DNT ( $60 \mathrm{ppb})$. Both films have similar thicknesses of about $40 \pm 5 \AA$.

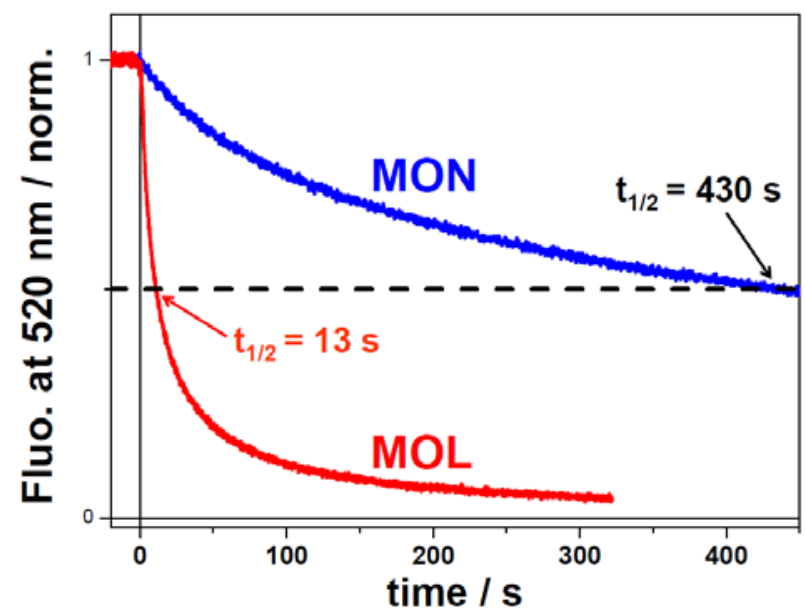

Fig.4 Time-dependent fluorescence intensity changes of MON and MOL films with similar thicknesses (i.e. $40 \pm 5 \AA$ ) exposed to a 300 $\mathrm{mL} \mathrm{min}^{-1} \mathrm{~N}_{2}$ flow with DNT at a vapor pressure of $\sim 60 \mathrm{ppb}$. Halftime for fluorescence quenching is also indicated for each film.

Whereas the fluorescence signal of MOL film decreases rapidly with a short quenching half-time of $13 \mathrm{~s}$ (see also Figure S6), the time-dependence for the fluorescence quenching of MON film is much more slower with a halftime $\left(\mathrm{t}_{1 / 2} \sim 430 \mathrm{~s}\right)$ more than one order of magnitude longer than that measured for MOL. In addition, the quenching efficiency after 30s DNT exposure is about $75 \%$ for MOL whereas it hardly reaches a value of $10 \%$ for MON film. Such a striking difference in the sensing time response should be confidently ascribed to the distinctive porosities within each film. Moreover, increasing the thickness of MOL film does not drastically affect its DNT sensing dynamics as compared to that observed for MON (see Figure S7). We clearly evidence the porogen role of the long alkyl side chain of MOL which appears as an efficient intermolecular spacer in solid state. This alkyl chain is thereby responsible for a lower density film which facilitates DNT diffusion as well as the analyte mass uptake. Reciprocally, it is worth stressing that the very slow sensing dynamics observed for MON film in presence of DNT vapors illustrates the fact that this $\pi$-conjugated material is perfectly configured to promote very dense sandwich-type supramolecular assemblies. Here, we show that a relatively simple structural change at the molecular scale which leaves invariant the electronic properties of the $\pi$ conjugated chromophore has a drastically impact on the sensing time response performances in thin solid film. In addition, similar sensing effects can be observed for other nitroaromatic analytes such 2,6 dinitrotoluene (2,6-DNT) as shown in Figure S8. Similar time-sensing responses as those measured for DNT can be derived which nicely generalizes our approach. In summary, we have developed a strategy to drastically accelerate the sensing dynamics for the detection of nitroaromatics on the basis of light-emitting molecule films. The key feature consists in incorporating a long flexible alkyl chain in the backbone of a $D-\pi-A$ alternated chromophore which is intrinsically configured to promote cofacial sandwich-type self-packing. The resulting antagonistic intermolecular interactions lead to a significant change of the film porosity with a subsequent faster DNT sensing whose detection dynamics is enhanced by more than one order of magnitude.

\section{Notes and references}

1 S. Shanmugaraju and P. S. Mukherjee, Chem. Commun., 2015, 51, 16014-16032.

2 D. T. McQuade, A. E. Pullen and T. M. Swager, Chem. Rev., 2000, 100, 2537-2574.

3 M. E. Germain and M. J. Knapp, Chem. Soc. Rev., 2009, 38, 2543-2555.

4 Y. Salinas, R. Martinez-Máñez, M. D. Marcos, F. Sancenon, A. M. Costero, M. Parra and S. Gil, Chem. Soc. Rev., 2012, 41, 1261-1296.

5 A. Rose, Z. Zhu, C. F. Madigan, T. M. Swager and V. Bulovic, Nature, 2005, 434, 876-879.

6 E. V. Verbitskiy, A. A. Baranova, K. I. Lugovik, K. O. Khokhlov, E. M. Cheprakova, M. Z. Shafikov, G. L. Rusinov, O. N. Chupakhin and V. N. Charushin, Dyes Pigm., 2017, 137, 360-371.

7 J.-S. Yang and T. M. Swager, J. Am. Chem. Soc., 1998, 120, 11864-11873.

8 Y. Geng, M. A. Ali, A. J. Clulow, S. Fan, P. L. Burn, I. R. Gentle, P. Meredith and P. E. Shaw, Nature Commun., 2015, 6, 8240.

9 M. A. Ali, S. S. Y. Chen, H. Cavaye, A. R. G. Smith, P. L. Burn, I. R. Gentle, P. Meredith and P. E. Shaw, Sens. Actuator B Chem., 2015, 210, 550-557.

10 M. A. Ali, S. Shoaee, S. Fan, P. L. Burn, I. R. Gentle, P. Meredith and P. E. Shaw, ChemPhysChem, 2016, 17, 3350-3353.

11 J. Li, C. E. Kendig and E. E. Nesterov, J. Am. Chem. Soc., 2007, 129, 15911-15918.

12 X. Yan, H. Wang, C. E. Hauke, T. R. Cook, M. Wang, M. L. Saha, Z. Zhou, M. Zhang, X. Li, F. Huang and P. J. Stang, J. Am. Chem. Soc., 2015, 137, 15276-15286.

13 B. Gole, W. Song, M. Lackinger and P. S. Mukherjee, Chem. Eur. J., 2014, 20, 13662-13680.

14 C. Madhu, B. Roy, P. Makam and T. Govindaraju, Chem. Commun., 2018, 54, 2280-2283.

15 J.-P. Malval, S. Achelle, L. Bodiou, A. Spangenberg, L. C. Gomez, O. Soppera and F. Robin-Le Guen, J. Mater. Chem. C, 2014, 2, 7869-7880.

16 S. Achelle, I. Nouira, B. Pfaffinger, Y. Ramondenc, N. Plé and J. Rodriguez-Lopez, J. Org. Chem., 2009, 74, 3711-3717.

17 F. J. M. Hoeben, P. Jonkheijm, E. W. Meijer and A. P. H. J. Schenning, Chem. Rev., 2005, 105, 1491-1546.

18 S. A. Jenekhe and J. A. Osaheni, Science, 1994, 265, 765.

19 N. Mataga, Y. Kaifu and M. Koizumi, Bull. Chem. Soc. Jpn., 1955, 28, 690-691.

20 E. Lippert, Z. Naturforsch., 1955, 10a, 541-545.

21 O. Yurchenko, D. Freytag, L. zur Borg, R. Zentel, J. Heinze and S. Ludwigs, J. Phys. Chem. B, 2012, 116, 30-39. 
22 K. Sreenath, C. V. Suneesh, V. K. Ratheesh Kumar and K. R. Gopidas, J. Org. Chem., 2008, 73, 3245-3251.

23 E. T. Seo, R. F. Nelson, J. M. Fritsch, L. S. Marcoux, D. W. Leedy and R. N. Adams, J. Am. Chem. Soc., 1966, 88, 3498-3503. 